\title{
Migrations internationales au prisme des rapports familiaux. Les familles sénégalaises à l'épreuve des refoulements des îles Canaries
}

International Migrations through the Prism of Family Relationships. Senegalese Families Difficult Experience of Migrants being Expelled from Canary Islands Migraciones internacionales desde la perspectiva de las relaciones familiares. Las familias senegalesas ante las devoluciones en frontera de las Islas Canarias

\section{Anaïk Pian}

\section{OpenEdition}

\section{Journals}

Édition électronique

URL : https://journals.openedition.org/remi/5530

DOI : 10.4000/remi.5530

ISSN : $1777-5418$

Éditeur

Université de Poitiers

Édition imprimée

Date de publication : 1 octobre 2011

Pagination : 77-100

ISBN : 979-10-90426-01-6

ISSN : 0765-0752

Référence électronique

Anaïk Pian, «Migrations internationales au prisme des rapports familiaux. Les familles sénégalaises à

l'épreuve des refoulements des îles Canaries », Revue européenne des migrations internationales [En ligne], vol. 27 - n² | 2011, mis en ligne le 01 octobre 2014, consulté le 16 avril 2022. URL : http:// journals.openedition.org/remi/5530 ; DOI : https://doi.org/10.4000/remi.5530 


\section{Migrations internationales au prisme des rapports familiaux. Les familles sénégalaises à l'épreuve des refoulements des îles Canaries}

\section{Anaik PIAN ${ }^{1}$}

$\mathrm{D}^{2}$ epuis quelques années, un certain nombre de travaux réalisés par des démographes, des anthropologues, des sociologues, des économistes et des géographes ont renseigné l'évolution des rapports familiaux au Sénégal. Les configurations familiales y sont appréhendées à travers l'étude des rapports conjugaux et de genre, des rapports intergénérationnels ou encore des stratégies résidentielles ; ces différentes dimensions se révélant étroitement imbriquées. De ces travaux, il ressort que, face à la persistance de la crise économique au Sénégal depuis les années 1980, les rôles et les rapports familiaux se renégocient, font l'objet de nouveaux compromis. Les mutations en cours, plus ou moins marquées, témoignent des modalités d'ajustement des acteurs sociaux confrontés à une précarisation s'étendant sur le long terme (Antoine et al., 1995).

Si cet article aborde la question des rapports familiaux au Sénégal, il le fait toutefois à travers une focale particulière, articulée à l'enjeu des migrations internationales. L'étude prend place dans un contexte bien précis : celui qui a conduit, en 20062007, de nombreux Sénégalais à risquer, depuis le Sénégal, la traversée clandestine vers les îles Canaries. Les Sénégalais qui tentent alors de rejoindre l'archipel espagnol en «cayucos » s'emparent du slogan suivant : « Barcelone ou la mort » (Barça ou Barzakh). Emblème de leur détermination à rejoindre l'Europe, cette expression qui s'est popularisée ne prend pourtant pas en compte une éventualité bien présente : celle de l'arrivée aux îles Canaries puis du refoulement par charter au Sénégal qui, pour l'année 2006, a concerné plus de 6000 Sénégalais $^{2}$. En effet, lorsqu'ils atteignent les îles Canaries après une traversée en mer de plusieurs jours, les intéressés, alors interceptés par la Guarda civil patrouillant au large des côtes canariennes, sont placés en centre de rétention. Ils y

1 Post-doctorante, Université de Bretagne Occidentale (ARS), avenue Duquesne, 29200 Brest ; anaikpian@yahoo.fr

2 Entretien à l'ambassade d'Espagne à Dakar mené en 2008. 
sont détenus une durée maximale de quarante jours, le temps que les autorités espagnoles déterminent leur identité et statuent sur leur sort. Ils sont refoulés vers leur pays d'origine si celui-ci parvient à être identifié et est signataire d'un accord ou d'un mémorandum de réadmission, ce qui en 2006 était le cas du Sénégal. Si leur identité ne parvient pas à être établie, les intéressés sont transférés sur le continent espagnol sans pour autant obtenir une régularisation de leur situation ${ }^{3}$.

Aussi, cet article s'intéresse à la dynamique des rapports sociaux - et notamment familiaux - qui ont entouré, en amont comme en aval, ce qui a été communément appelé le «phénomène des pirogues ». Quels sont les impacts de ces refoulements sur les rapports familiaux et, plus largement, sur la vie des quartiers très touchés par les départs en pirogues vers les îles Canaries ? Ces départs et les refoulements vers le Sénégal peuvent, nous semble-t-il, être analysés comme un phénomène social qui, tout à la fois, renseigne sur l'ordre local et a des effets sur lui ${ }^{4}$.

Notre enquête ${ }^{5}$ 'est étendue sur deux périodes, la première en février-mars 2008, la seconde en juin-juillet 2008. Dans une perspective qualitative, nous avons fait le choix d'un terrain à petite échelle. Celui-ci s'est principalement déroulé à Mbour, port de pêche de la petite côte situé à quatre-vingt-cinq kilomètres de la capitale sénégalaise, et à Yarakh, quartier de pêcheurs de la banlieue de Dakar ${ }^{6}$. Nous avons également rencontré quelques Sénégalais résidant à Cayar ainsi qu'au centre de Dakar. Nous avons effectué des entretiens approfondis avec vingt-et-un refoulés côtoyés dans la durée et revus, pour la majorité d'entre eux, entre notre premier et second terrain. En complément des entretiens, nous avons privilégié des discussions informelles ainsi que des observations sur les lieux de vie et de travail des intéressés. L'établissement de relations de confiance dans la durée et la possibilité d'effectuer des récits croisés avec leurs proches ont permis de déjouer les pièges méthodologiques liés à un sujet très médiatisé à propos duquel domine un certain nombre de discours stéréotypés. Lorsque le sociologue arrive sur le terrain, il est tout d'abord renvoyé à l'image du journaliste qui filme des témoignages contre rémunération dans un laps de temps balisé, à moins qu'il ne soit assimilé à un expert du développement, souvent accusé de proposer des aides sans suite. Sur vingt-et-un refoulés rencontrés, huit ont tenté la traversée vers les îles Canaries deux

3 D'autres déposent une demande d'asile ou peuvent obtenir un permis de séjour pour « raisons humanitaires ", notamment si le retour au pays est impossible. Dans d'autres situations encore, les migrants atteignant les îles Canaries peuvent être refoulés vers le pays de départ à partir duquel ils ont tenté la traversée, même s'il ne s'agit pas de leur pays d'origine. Le Maroc a signé avec l'Espagne de tels accords impliquant la réadmission de ressortissants de pays tiers ayant transité par le territoire marocain. Cet article, toutefois, ne traite pas de tels parcours migratoires. Cf. sur ce point Pian (2009a). Pour plus de données sur la situation migratoire aux îles Canaries et une revue de la littérature existante sur le sujet, cf. les rapports de Marreo (2008) et de Godenau et Hernadez (2007). Si ce dernier rapport traite plus spécifiquement de la migration irrégulière à Tenerife, il n'aborde pas toutefois, en tant que tel, la situation des migrants arrivés en « cayucos », mais porte plus particulièrement sur le cas des étrangers sans-papiers vivant sur l'île.

4 Nous ne traiterons pas ici des associations de refoulés qui ont fait l'objet d'un autre article (Pian, 2011).

5 Réalisée dans le cadre du projet ANR Mitrans, " Migrations de transit en Afrique » mené avec l'Urmis, l'Urmis-Soliis et l'Ifas. Cette réflexion a donné lieu à une première communication au CEPED en avril 2010. Par ailleurs, les noms des enquêtés ont été changés.

6 Il faut signaler, à Yarakh, la fermeture d'une usine de glace il y a plusieurs années déjà, et qui a eu de fortes incidences socio-économiques, de nombreux habitants du quartier se retrouvant au chômage. 
fois de suite, après un premier refoulement ou un naufrage au large des côtes marocaines ou mauritaniennes. Les deux-tiers de notre corpus sont constitués d'hommes mariés et de pères de famille ${ }^{7}$. Si leur moyenne d'âge peut être arrondie à trente-trois ans, les refoulés de Mbour présentent un profil plus âgé que ceux de Yarakh. Alors qu'à Mbour, la plupart d'entre eux sont pêcheurs et mareyeurs ${ }^{8}$, à Yarakh, les Sénégalais rencontrés sont mécaniciens, apprentis-menuisiers, grutiers, étudiants ou sans emploi. Parmi eux, un seul avait déjà tenté de rejoindre les îles Canaries à partir du Maroc en 2004. Notre corpus ne prétend pas à une représentativité sur le plan statistique 9 . En revanche, il s'inscrit dans une conception ethnographique de l'individu porteur et révélateur d'une histoire sociale et individuelle (Beaud et Weber, 2003). Comme le rappelle Jounin (2008), la problématique statistique est déplacée lorsque la recherche s'intéresse d'abord et avant tout à l'analyse de relations sociales appréhendées comme un construit social dans un contexte donné. Notre démarche privilégie ainsi un angle de vue qui, certes, n'épuise pas la complexité du réel, mais porte un éclairage sur un aspect de ce phénomène migratoire.

\section{FOCUS SUR LES RAPPORTS FAMILIAUX EN AMONT DU DÉPART}

Pour comprendre la manière dont les refoulements des îles Canaries ont affecté l'institution familiale, la dynamique des rapports sociaux au moment du départ doit tout d'abord être éclairée. La rivalité familiale d'une part, et la prise en compte du volet relationnel de la précarité d'autre part, sont deux dimensions qui, à ce titre, prennent toute leur importance.

\section{La marginalisation sociale, volet relationnel de la précarité}

La rivalité familiale et, en premier lieu, la rivalité entre coépouses est une réalité sociologique qui traverse de nombreuses familles sénégalaises dans un contexte de crise économique et de restructuration des rapports sociaux autour d'une tension croissante entre solidarité communautaire et processus d'individualisation (Marie, 1997). L'allongement de la cohabitation intergénérationnelle ${ }^{10}$, qui constitue une forme de réponse des familles à la crise, n'atténue pas, loin s'en faut, ces rivalités. Certes, les cohabitations permettent la mise en commun de certaines ressources et dépenses, notamment celles liées au loyer et aux charges apparentées (électricité, eau, etc.). Toutefois, même lorsque la famille élargie vit sous le même toit, chaque lignée tend à entretenir ses projets de promotion

7 Des femmes et des mineurs ont également emprunté les embarcations vers les îles Canaries même si nous ne traitons pas ici de cette population.

8 Ce qui tient au fait que nous avons mené notre terrain au niveau du port de pêche.

9 À partir des données répertoriant le profil des personnes déférées pour « émigration clandestine » devant la justice sénégalaise et plus précisément devant les parquets de Saint-Louis, Dakar, Thiès et Ziguinchor, Gonin et Robin (2009 : 159) établissent les statistiques suivantes : plus de $95 \%$ des intéressés sont des hommes et $60 \%$ d'entre eux ont entre vingt-cinq et trente-neuf ans (avec une moyenne d'âge totale d'environ vingt-huit ans). Par ailleurs, $93 \%$ d'entre eux occupaient une activité professionnelle avant leur départ, principalement dans les domaines de la pêche, du commerce, de l'artisanat et des transports.

10 Particulièrement marqué à Dakar en raison du coût élevé du logement. Pour plus de précisions, voir Antoine (2007) et Diagne et Lessault (2007). 
sociale. En dépit de l'existence d'un salon ou d'une pièce commune ${ }^{11}$, les chambres sont par excellence les lieux où chacun cherche à accumuler des biens propres : télévision, belle armoire, chaînes Hi-fi, etc. En outre, dans les situations de polygamie, encore très courantes y compris en milieu urbain comme à Dakar ${ }^{12}$, les coépouses tendent à pratiquer des « comportements budgétaires individualisés » (Dial, 2007) en conservant à des fins personnelles leurs ressources financières propres, acquises par elles-mêmes ${ }^{13}$ ou par l'intermédiaire de leurs enfants. Bien que l'institution polygame soit régie par des règles bien définies, censées garantir l'équité entre les coépouses, la concurrence entre celles-ci peut être prégnante. La polygamie s'organise selon un système de rotation qui s'applique d'une part, à la répartition de certaines tâches domestiques, et d'autre part, à l'entretien des relations sexuelles avec le mari. Ainsi c'est à tour de rôle que les épouses sont amenées à préparer le plat du jour et, conjointement, à dormir avec l'époux. Or, la cuisine est un moment privilégié où se mesure la rivalité entre coépouses, chacune essayant de faire « mieux » que l'autre pour plaire au mari, ce qui suppose pouvoir acheter les « meilleurs » aliments. Dans la mesure où celui-ci est tenu de remettre à chacune de ses femmes une même dépense quotidienne, ces dernières peuvent, le cas échéant, compléter la somme attribuée en mobilisant leurs propres ressources.

Les rivalités familiales peuvent également s'étendre entre voisins ou entre frères d'une même fratrie. Guérin (2008) montre comment les familles sénégalaises conjuguent de manière tensionnelle espace de coopération (mise en commun des ressources) et espace de conflits (affirmation individualiste) donnant lieu à diverses formes de compromis et de négociation. Cet état de fait transparaît bien dans les propos d'un jeune de Yarakh, Cissé. Célibataire et âgé de vingt-cinq ans, Cissé est orphelin de mère depuis l'enfance. Il a tenté la traversée vers les îles Canaries durant l'été 2006, alors qu'il était étudiant en première année d'anglais à l'université de Dakar et qu'il devait repasser des examens de rattrapage. Faisant allusion à sa situation familiale (il vit dans la maison de la lignée paternelle), il souligne : "Ici, on ne vit pas en famille, mais seulement en voisins. Moi, je peux rester un mois sans entrer dans la chambre de ma tante ».

La rivalité familiale se double de la dépréciation sociale de ceux qui, se situant à la marge, ne sont pas en mesure de subvenir aux besoins du foyer, qu'ils soient étudiants, sans-emploi ou que leur activité rapporte trop peu. Certes, dans le cadre des cohabitations familiales élargies, l'usage s'appuie sur le principe du talqal, que Diagne et Lessault (2007) définissent comme une sorte de « bricolage» reposant sur la compilation de petits revenus

$11 \mathrm{Au}$ décès d'un personnage fédérateur, incarnant l'autorité traditionnelle comme un grand-père, ces pièces communes disparaissent parfois.

12 Comme le souligne Dial (2007), un ensemble de facteurs sociaux, économiques et culturels favorisent le maintien de la polygamie. Reprenant la classification établie par Antoine et Nanitelamio (1995) l'auteure montre comment celle-ci peut relever de plusieurs types. La « polygamie du pauvre » correspond ainsi aux situations dans lesquelles le mari compte sur la participation financière de ses épouses à la vie du foyer ; à l'opposé, se donne à lire la «polygamie ostentatoire » qui permet d'afficher sa réussite sociale. Entre ces deux pôles, se situent la "polygamie imposée » (par la famille) et la polygamie de " retour » qui concerne souvent des intellectuels effectuant un « retour » vers des valeurs religieuses. Dial (2007) relève l'importance de la "polygamie du pauvre » dans son enquête de terrain à Dakar et note que celle-ci intervient souvent tardivement dans la vie matrimoniale des hommes.

13 Cf. plus loin. 
apportés par les différents membres du ménage. Cependant, la participation des jeunes au budget familial demeure le plus souvent irrégulière, en raison de leur situation précaire sur le marché de l'emploi. À Dakar, $30 \%$ des adultes âgés de vingt-cinq à vingt-neuf ans sont inactifs ou au chômage, tandis que dans 60 \% des ménages dirigés par un chef de famille de plus de cinquante-cinq ans, se trouve au moins un enfant adulte sans emploi (Antoine, 2007 : 39). Or, la dépendance financière déteint sur la légitimité et l'honneur social : à tel point d'ailleurs, que certains jeunes rencontrés à Yarakh usent de tactiques de contournement (Goffman, 1991) en s'endettant auprès des boutiquiers du quartier pour pouvoir, de temps à autre, répondre aux sollicitations faites sans trop «perdre la face ». Ces tactiques participent d'une même logique de débrouille que celles décrites par Diagne et Lesssault (2007), même si elles peuvent prendre des formes inversées. En effet, les auteurs font part, a contrario, de la situation de jeunes actifs qui, à Dakar, s'attachent à limiter leur présence dans l'espace domestique afin d'éviter d'être constamment sollicités pour de petites dépenses quotidiennes.

Ces situations de marginalisation sociale, où la position socio-économique influe sur la place conférée dans la famille ${ }^{14}$, se retrouvent aussi bien parmi les jeunes de Yarakh rencontrés que parmi des pêcheurs de Mbour, pères de famille proche de la quarantaine.

Cissé, étudiant, fait ainsi part de sa mise à l'écart au sein de la famille :

"- Parfois, je me réveille et je n'ai pas de quoi prendre le petit-déjeuner, ou je rentre chez moi et je trouve le repas fini, on ne m'a rien gardé et je n'ai pas d'argent... Ici c'est dur, il y a des familles qui te rappellent chaque jour que tu n'as pas de travail...

- Mais tu faisais tes études?

- Eux, ils ne voient pas ça comme ça, ils ne croient pas que tu puisses trouver un travail avec mon diplôme ${ }^{15}$. Et personne ne m'aide pour financer mes études, de toute façon je n'avais plus les moyens de continuer. Moi pourquoi je suis parti? Pour avoir quelque chose et montrer à ma famille que je suis capable. Un jour, la femme de mon oncle m'a mal parlé. Elle ne m'avait pas gardé de repas. J'ai failli me battre avec elle. Ici, si tu ne travailles pas on ne te respecte pas. Si tu frappes un petit enfant dans la maison on te dit : "Pourquoi tu le frappes?" Tu ne participes pas aux débats, on ne te demande pas ton avis si tu n'as pas d'argent ».

Modou, quant à lui, est âgé de quarante ans et travaille comme pêcheur à Mbour depuis vingt ans. Il fait part de ses difficultés, accentuées depuis la flambée du prix du carburant ${ }^{16}$, à satisfaire les charges qui lui incombent. Des tensions quotidiennes le confrontent à la femme de son frère cadet qui vit en France depuis plusieurs années :

"Si tu n'as pas d'argent, on te traite de vaurien. Même dans ta famille on ne te respecte pas. Même ta femme et tes enfants. Si tu n'as pas les moyens, si tu ne peux pas les éduquer, tu n'as pas d'autorité sur eux [...]. Ici le bon choix, c'est d'avoir de

14 Si la dépréciation sociale peut être perçue comme étant de l'ordre du jugement, la marginalisation sociale est avant tout de l'ordre de la pratique.

15 Cela est aussi peut-être accentué par le fait que Cissé n'a pas d'emblée réussi ses examens de première année.

$16 \mathrm{Au}$ printemps 2008, plusieurs manifestations contre l'augmentation du prix de l'essence ont lieu dans différentes villes du Sénégal. Il y a même pénurie. 
l'argent. Tu n'en as pas, tu n'es plus rien. Tu n'as pas de parole. Un homme qui n'a pas de parole n'est rien. On ne t'écoute pas. Même si tu es l'aîné. Mais si c'est le petit frère qui a de l'argent qui dit quelque chose, tout le monde l'écoute».

En dépit de leur différence d'âge et de statut matrimonial, les situations décrites par Modou et Cissé font état d'un malaise partagé. Les schèmes de leur discours se construisent autour d'une opposition sémantique commune entre « argent et parole » d'un côté, et « rien » ou " défaut de reconnaissance et de respect» de l'autre. Les moyens financiers (re)définissent les hiérarchies familiales à travers l'autorité conférée et la précarité économique dépossède du rôle d'aîné dans la fratrie, y compris pour les hommes mariés. Pour Cissé, qui n'est pas encore marié et vit avec sa famille dans la maison du grandpère paternel (aujourd'hui décédé), le fait de se retrouver dans une situation de dépendance intergénérationnelle, à la fois financière et résidentielle, le place dans une position inconfortable, douloureusement vécue. Diagne et Lessault (2007 : 29) rappellent à ce titre la force du proverbe wolof selon lequel « le garçon doit prendre la relève de son père lorsqu'il a les mêmes capacités physiques que lui » (gor bün mün sol dalu bayam wutu ko warnako). Cissé se retrouve ainsi dans une situation proche de la conception mertonienne de l'anomie (Merton, 1953) dans la mesure où il doit faire face au décalage entre les objectifs et les valeurs mises en avant par la société, et les moyens existants pour y répondre.

Modou, quant à lui, se situe dans la position de ces pères de famille dont la légitimité traditionnelle est diminuée au regard de la difficulté à assumer la prise en charge financière du ménage. Cette situation n'est pas un cas isolé, comme en attestent les travaux de Coumba Diop (2009) et de Dial (2007) qui évoquent la figure, de plus en plus fréquente, de ces pères « économiquement humiliés ».

Pour les plus jeunes, l'image de ces pères dépassés par leurs charges familiales (d'autant plus lorsqu'ils atteignent un âge avancé) constitue une figure repoussoir à laquelle ils ne veulent s'identifier. Ce qu'ils craignent avant tout, c'est de se retrouver un jour dans une même situation. Antoine (2007) a souligné la position difficile de ces aînés qui, lorsqu'ils arrivent à l'âge de la retraite et commencent à avoir des problèmes de santé liés à la vieillesse, ont encore des enfants à charge ${ }^{17}$.

Enfin, à l'instar de Cissé, de nombreux jeunes sans revenus font part des difficultés à avoir de bonnes relations avec les filles de leur âge. Selon eux, en effet, celles-ci chercheraient avant tout des petits copains capables de les entretenir. Dans ses travaux, Fouquet (2008) qui s'intéresse à l'imaginaire migratoire des Sénégalais, rapporte également plusieurs témoignages montrant la difficulté, pour les jeunes sans ressources, à se positionner sur le marché matrimonial. L'on voit ici, comment la matérialité économique de la précarité ne prend tout son sens que replacée dans la dynamique des rapports sociaux qui s'y rapportent. Dans ces conditions, tenter la traversée vers les îles Canaries est apparu pour les refoulés rencontrés, comme un moyen de (re)conquête possible d'un statut : «je voulais leur montrer que j’étais capable » appuie Cissé.

17 L'écart de l'âge au mariage entre les hommes et les femmes explique en partie ces situations. À Dakar, l'écart de l'âge au mariage entre les hommes et les femmes est, pour les hommes âgés de plus de cinquante-cinq ans, de dix-huit ans. Il est de neuf ans pour les nouvelles générations. Antoine (2007 : 37). 
Mais, pour saisir toute la déclinaison des rapports familiaux au moment du départ, une autre dimension encore doit être prise en compte. Certaines mères se sont fortement mobilisées dans le financement des voyages, n'hésitant pas à y investir leurs économies, leur tontine ou à vendre leurs bijoux ${ }^{18}$. Cet investissement, à la fois moral et financier, est à replacer dans le cadre des rapports sociaux, matériels et idéels, qui traversent les familles sénégalaises. Tout d'abord, il faut rappeler que la division sexuée des fonctions au sein des familles sénégalaises a connu des évolutions, les femmes prenant de plus en plus en charge les dépenses quotidiennes (Guérin, 2008) ${ }^{19}$ et non plus seulement les besoins exceptionnels (liés aux mariages, baptêmes, etc.). Pour ce faire, elles mobilisent des circuits financiers spécifiquement féminins (tontines), tandis qu'une part croissante d'entre elles entretient de petits commerces de rue (vente de noix de cajous, de poissons, etc.). D'une manière générale, elles sont aussi, depuis quelques années, plus nombreuses sur le marché de l'emploi (Dial, 2007). Or, au rôle économique des femmes dans les ménages se conjugue une dimension symbolique de non moindre importance. Les représentations populaires, en effet, attribuent aux mères la responsabilité de la réussite sociale de leurs enfants : ce qui est alors jugé dans le regard d'autrui, ce n'est pas tant leur qualité de mère, que leur qualité d'épouse. Une femme dont l'enfant ne réussit pas socialement est renvoyée à l'image d'une mauvaise épouse, n'ayant pas su prendre en charge son foyer ni faire preuve d'un comportement exemplaire auprès de son mari. La norme, en la matière, se mesure au regard de l'abnégation de l'épouse (c'est-à-dire sa soumission) et sa capacité à satisfaire sexuellement son époux. Cette «symbolique du travail de la mère », qui renvoie à l'adage wolof « lig éeyu ndey aňub doom », est très présente dans les mentalités collectives. Elle est d'ailleurs relayée par de nombreuses chansons populaires, à l'image de «Sēy » (" le mariage ») chanté par Ndongo Lō (Dial, 2007). Ainsi, dans ce contexte où s'entremêlent dimensions socio-économiques et symboliques, certaines mères n'ont pas seulement financé le voyage de leur fils vers les îles Canaries : elles ont pu les convaincre du bien-fondé de l'entreprise. Il faut dire en effet que, peu à peu, les traversées en pirogues ont soulevé un fort engouement collectif.

\section{L'engouement collectif}

À Yarhah et à Mbour, les départs en «cayucos » vers les îles Canaries ont suscité un véritable phénomène d'engouement bien que des Sénégalais, y compris des pêcheurs et capitaines de pêche, se soient tout d'abord montrés sceptiques quant à la possibilité de pouvoir rejoindre l'archipel espagnol à partir des côtes sénégalaises. En témoignent les propos d'Aziz qui, âgé de trente-deux ans, travaille comme mareyeur à Mbour depuis huit ans :

"Ça fait longtemps que j'avais l'idée de l'Europe dans ma tête, et quand j'entends les gens qui prennent les pirogues pour aller en Espagne, je me dis que ça, c'est impossible. Mais les gens me disent que c'est vrai. Deux ou trois semaines après, il y a des copains qui travaillent ici avec moi qui ont téléphoné pour me dire

qu'ils sont en Espagne. En Espagne? Mais comment tu as fait? Tu vas prendre une pirogue et payer 400000 ou $500000 \ldots$ C'est pas vrai! Si! Imagine-toi. Cela

18 Sur le rôle des mères, voir également Bouilly (2008).

19 On note également, ces dernières années, une augmentation du nombre de ménages dirigés par des femmes. En 2001, c'est le cas de 19,4 \% des ménages au Sénégal (Charbit et Kebe, 2007). 
fait des années que je rêve de ça. Si un de mes copains a pu partir par ce moyen, pourquoi pas le faire! $»^{20}$.

Aujourd'hui, le secteur de la pêche n'attire plus les jeunes générations, tandis que les aînés déplorent une activité de moins en moins rentable. En ligne de mire, sont mis en cause les accords de pêche qui, signés avec l'Espagne ${ }^{21}$, autorisent des chalutiers espagnols à pêcher dans les eaux territoriales sénégalaises. Il n'en demeure pas moins que la modernisation - notamment en termes d'équipements du secteur de la pêche - a facilité, comme le rappellent Sall et Morand (2008), les traversées vers les îles Canaries. Mais c'est aussi l'expérience des pêches « au long cours » qui est mise à profit. À Mbour, par exemple, des capitaines de pêche partent régulièrement pêcher en Gambie voire en Guinée, ce qui implique des expéditions d'une dizaine de jours, soit une durée équivalente à la traversée vers les îles Canaries ${ }^{22}$ (sauf avatars rencontrés sur la route).

Aussi, au fur et à mesure que les premiers partis atteignent les îles Canaries puis téléphonent des centres de rétention situés sur l'archipel espagnol, et que certains, transférés sur le continent, joignent leur famille pour relayer la nouvelle, la tentation de la traversée clandestine gagne un nombre croissant de Sénégalais. C'est ainsi que Diagne bénéficiant pourtant d'une situation sociale privilégiée - sa mère adoptive étant sénatrice tente lui aussi le voyage avant d'être refoulé au Sénégal. Sayad (1999) a montré comment l'entretien d'un « mensonge collectif » contribue à auto-alimenter une émigration. L'on retrouve ici un processus en certains points similaires. Les Sénégalais parvenus aux îles Canaries encouragent leurs camarades à les rejoindre sans les informer des véritables conditions du voyage : " c'est pas grave » ont-ils coutume de dire. Les refoulés, quant à eux, racontent rarement, de retour, la réalité de la traversée qui demeure un sujet tabou, indicible et non racontable à ceux qui n'ont pas tenté l'expérience. Ils ne veulent pas, de surcroît, inquiéter les familles de camarades se trouvant en haute mer. Et, s'ils tentent de dissuader des proches de tenter le voyage, ils sont souvent accusés d'égoïsme et renvoyés à l'image négative du refoulé qui, n'ayant pas atteint son objectif, ne veut pas être doublé par d'autres. La dynamique des rapports sociaux participe ainsi de la construction du «mensonge collectif», dès lors qu'elle laisse peu d'alternatives aux refoulés : par d'éventuelles mises en garde, ces derniers risquent surtout d'accroître la disqualification qui les menace, du fait de la mise en échec de leur tentative d'émigration. Le phénomène d'engouement, en outre, est partagé par certaines familles qui perçoivent dans cette nouvelle route migratoire un moyen inespéré de promotion sociale.

Fatou, institutrice dans une école publique, est mère de trois enfants dont l'aînée a huit ans. Bien que son mari ait effectué des études supérieures, il n'a pas de travail fixe.

20 Parmi les refoulés rencontrés, quelques-uns avaient auparavant envisagé d'emprunter la route marocaine pour se rendre en Europe. La plupart, cependant, avaient écarté ou n'avaient pas songé à tenter un parcours si long, ponctué de nombreux détours. Se détachant de la conception de l'aventure et de l'aventurier (Pian, 2009a), ils ont en revanche saisi ce qui est apparu comme une opportunité inédite, celle d'une voie rapide et directe leur permettant de rejoindre l'Europe : «Marcher comme ça par le Maroc ? Non, ça c'est grave... Et ça prend du temps, tu ne sais pas quand tu vas arriver. Et puis financièrement, tu ne peux pas arriver à t'en sortir car il faut plus d'argent. Plus le voyage est long, plus ça demande de moyens » souligne ainsi un jeune de Yarakh.

21 Comme plus largement avec les pays européens.

22 Même si les courants sont plus forts pour se rendre jusqu'à l'archipel espagnol.

REMI 2011 (27) 2 pp. 77-100 
La famille loue une chambre à Pikine pour 25000 CFA par mois. Fatou est inquiète pour l'avenir, elle pense aux frais de scolarité pour ses filles, aux factures à payer et se demande combien de temps encore la famille pourra vivre dans une seule pièce faisant office de salon, de chambre à coucher et de cuisine, avec un petit réchaud à gaz posé dans un coin :

"Modou c'est mon petit frère... Je l'ai aidé à partir en lui donnant ma tontine, 300 000. Lui, il avait 200 000. On avait discuté avec mon père. Je l'avais encouragé à partir car on n'avait pas les moyens. Et Modou avait pitié de moi... Si Modou était en Europe, je crois qu'il pourrait m'aider, aider mon père. C'est pourquoi j'ai fait ce sacrifice pour lui. Et je ne savais pas que ça se passait comme ça. C'est après que j'ai vu à la télé, car Modou était dans les premiers à partir».

Cet engouement collectif est à replacer dans les conditions de vie sociétale, dont participent la dynamique des rapports familiaux, ainsi que le contexte de crise économique et, plus largement, de rupture de confiance dans l'État sénégalais. Peut-être faut-il rappeler que la dévaluation du franc CFA en 1994 a entraîné une baisse du pouvoir d'achat des familles sénégalaises sans pour autant permettre une réduction importante du taux de chômage qui, aujourd'hui encore, est élevé, y compris parmi les jeunes diplômés (Daffé et Dansokho, 2003). En 2001, le Sénégal est d'ailleurs classé parmi les PMA (Pays les Moins Avancés) par le Conseil économique et social de l'ONU et l'alternance politique marquée par l'élection de Wade aux présidentielles de mars 2000, après quarante ans de gouvernance socialiste, n'a pas été à la hauteur des changements attendus par une grande partie de la population. Les déceptions sont nombreuses sur les plans socio-économiques comme de l'assainissement de la vie politique (Dahou et Foucher, 2005). À côté des inégalités sociales persistantes, voire croissantes, de nombreux points noirs (malversations financières, connivences avec certains hommes d'affaires, pressions, voire violences, à l'encontre de certains opposants politiques ou de personnalités religieuses, etc.) entachent la légitimité de Wade, pourtant réélu en 2007. En 2008, le Sénégal est également pointé du doigt par plusieurs bailleurs de fonds qui conteste la gestion financière des pouvoirs publics (Diop, 2009) tandis qu'une forte contestation sociale, notamment alimentée par l'augmentation des prix des denrées alimentaires et des carburants, prend forme dans le pays.

Plus qu'une quelconque rationalité à proprement parler, ces départs en pirogues mettent alors en scène un projet d'agir au sens où l'entend Schutz (1994). Ce projet d'agir, qui évalue les possibilités du futur après-action, se construit en comparaison avec les conditions de vie présentes et l'horizon que celles-ci entrouvrent in situ ${ }^{23}$. Ces « attentes subjectives » prennent aussi appui sur l'exemple des émigrés vivant dans les pays occidentaux et qui, par leurs transferts de fonds, leur investissement immobilier au Sénégal

23 «Tout projet consiste en une anticipation du futur sur le mode de l'imagination » (Schutz, 1932, cité in Cefaï, 1994 : 119). Le premier pas de l'agir est la visualisation de la situation désirée, une fois que l'action anticipée aura été accomplie et sera révolue. L'agir prend sa source dans la pré-vision de l'action achevée, il se pro-jette dans un à-venir-déjà-passé, qui s'indique au futur antérieur (...) comme le pôle téléologique vers lequel vont s'unifier et s'orienter les phases de l'agir. Le projet d'agir propose des « représentations intuitives » de l'action « comme si » elle était déjà accomplie, il suppose l'imagination d'un déroulement d'action avant le passage à l'acte, et n'est pas impliqué dans le cours même de l'agir (...). Le projet d'agir est une remémoration « avant coup » de l'action réalisée « après coup » (Cefaï, $1994: 119)$. 
ou encore à l'occasion des vacances passées au Sénégal, incarnent la figure de l'ascension sociale (Pian, 2009a).

\section{Négociations et temporalités du départ}

Il reste que la négociation du départ au sein des familles a pris des formes variées. Certains optent pour le départ incognito. D'autres disent avoir prévenu quelques membres de leur famille seulement, souvent leur épouse ou leur mère. La décision et la préparation du voyage peuvent faire l'objet de concertation et de débats, comme le montre ci-dessus le témoignage de la sœur de Modou. En 2004, Abdou, quant à lui, a rejoint les îles Canaries à partir des côtes marocaines avant d'être refoulé au Sénégal. Lorsque son frère cadet fait part à sa famille, en 2006, de son intention d'emprunter les pirogues, ses parents préfèrent miser sur son expérience et, à cette fin, son père vend une partie de sa récolte. D'autres Sénégalais ont vendu leur matériel de travail, pirogues, moteurs, pièces détachées ou encore du mobilier pour financer leur voyage. En dépit de leur rôle majeur, les femmes n'ont pas été seules à investir dans la traversée et, parfois, un frère a mobilisé ses quelques biens pour compléter la somme d'argent nécessaire.

Les temporalités dans la préparation du départ se déclinent également sur des échelles variées. La plupart ont soigneusement préparé leur traversée en consultant un marabout pour s'armer mystiquement ${ }^{24}$. Le marché du mysticisme participe de l'économie de la migration (Pian, 2009a) ${ }^{25}$. Gris-gris, sacrifices et bénédictions font partie des préparatifs. À cela s'ajoutent l'achat de cigarettes et, souvent, de l'alcool, des oranges ou encore de la « vache qui rit $»^{26}$. Les plus avertis se munissent d'amphétamines. D'autres partent dans la précipitation. Des pêcheurs ont pu être sollicités en dernière minute pour faire partie de l'équipage. En effet, les pêcheurs les plus expérimentés, en premier lieu desquels les capitaines de pêche, peuvent être recrutés comme capitaine de pirogue et, ce faisant, ils sont exonérés du paiement de la traversée, dont le prix est fixé entre 400000 et 500000 CFA. Ils sont suppléés par des membres d'équipage qui, en fonction de leur attribution, bénéficient de réduction importante sur le prix du voyage, voire d'un passage gratuit. Lorsque ces offres se présentent à la hâte, sans avoir été programmées à l'avance, certains préfèrent cependant les décliner, n'osant défier la mer sans avoir préalablement consulté leur marabout. D'autres, encore, présents sur la plage au moment où se prépare un départ, embarquent sur « un coup de tête $»^{27}$. C'est le cas de Cissé :

24 Si la plupart des passages se préparent mystiquement, la barque elle-même est parée de grisgris. Certains d'entre eux sont censés la rendre invisible en vue d'éviter leur interception par les patrouilles maritimes dans les eaux territoriales sénégalaises. À l'approche des îles Canaries, ces derniers doivent être enlevés au risque que le capitaine et les passagers passent à côté de l'archipel sans le voir.

25 Ce fait est également particulièrement marqué dans l'aventure par le Maroc, à partir duquel d'autres Sénégalais tentent de rejoindre l'Europe (Pian, 2009a).

26 Des repas communs sont en outre préparés sur un réchaud à l'intérieur de la pirogue, constitué de riz cuit à l'eau douce.

27 Une première pirogue de taille moyenne, pouvant comporter une vingtaine de personnes, se chargeait d'aller chercher les passagers sur les plages pour les conduire à un « cayucos » beaucoup plus grand qui attendait arrimé au large, pour déjouer les contrôles policiers. 
"Après ma dispute ${ }^{28}$ avec ma tante, je suis resté trois jours sans venir chez moi. Et moi je ne savais pas quoi faire exactement, je me disais que je devais chercher du travail pendant ces vacances. Je me posais trop de questions, qu'est ce que je dois faire. Un dimanche, on est parti dans la boîte de nuit. On s'est bien défoulé.

Et la nuit on est revenu ici faire du thé chez moi, dans la petite chambre. Et on parlait toujours des problèmes des meufs... On ne pensait même pas partir en mer, on avait des copains qui étaient partis depuis longtemps, et nous on avait même les moyens d'y aller... [...] Et là, on voit une pirogue qui part. Les clients étaient en train de monter. Un copain nous a dit: "Eh, les gars, on ne va pas rester ici les bras croisés". [...] Donc on s'est dit, on devait partir, et Inchalla il n'y aura pas de problème sur la mer, Dieu est grand. On disait on va partir, pas de problème, en groupe, et on va être là-bas ensemble... tout ça quoi... On était en train de rêver quoi! Et on est parti le jour même, même pas trente minutes après ! [...] On est venu pour monter et les gens de la pirogue sont venus avec des couteaux, des haches... il y avait même un pistolet. Mais, eux, ils ne sont pas cons. Ils savent que s'ils tuent quelqu'un ici, et que la pirogue a des problèmes et qu'ils vont retourner, il y aura des témoins pour les dénoncer. Les clients, on les connaissait, mais pas les capitaines. On s'est bagarré, bagarré. Après seulement moi et trois copains on a réussi à monter. Et on savait qu'une fois dans la pirogue, ils ne vont pas nous dire de sauter, sinon, nous, on va emmener des problèmes dans la pirogue et ça, c'est pas bon pour ce genre de voyage... ».

Quelques jours avant le départ pour ceux qui l'ont programmé, à moins que cela soit au moment même de l'embarquement ou durant la traversée, il n'est pas rare que des doutes sur le choix effectué s'emparent des intéressés. Mais, même si le départ n'a pas encore eu lieu, il leur est difficile de renoncer, d'autant plus s'ils se sont endettés. Leurs regrets et le sentiment d'avoir été dupé sur les conditions de la traversée n'empêcheront pas, toutefois, certains de tenter leur chance une seconde fois, après avoir été refoulés des îles Canaries. Durant la traversée à destination de l'archipel espagnol, les intéressés peuvent être entassés jusqu'à une centaine dans les «cayucos ». En dépit des aménagements généralement réalisés au sein des embarcations (bâche abritant un réchaud destiné à faire cuire du riz, etc.) le voyage confronte à la déshydratation, la faim, les nausées et plus largement aux aléas climatiques. Et, même si les « cayucos » parviennent à atteindre les îles Canaries, certains passagers décèdent durant la traversée tandis que d'autres sombrent dans des états proches de la folie ${ }^{29}$. En 2006, 600 cadavres ont été retrouvés au large des côtes canariennes tandis que le nombre total de disparus entre les îles Canaries et les côtes ouest-africaines est estimé à plus de 6000 selon un responsable des services d'immigration espagnol (Blanchard, Clochard et Rodier, 2008). Selon ces mêmes auteurs, le renforcement des patrouilles dans cette zone maritime au cours de l'année 2006 n'a fait qu'accentuer les risques encourus par les candidats à l'émigration qui, dès lors, empruntent des itinéraires de plus en plus dangereux. Ainsi, si en 2007, le nombre d'arrivées irrégulières en « cayucos » aux îles Canaries a fortement baissé par rapport à l'année précédente, en revanche, le nombre de corps sans vie retrouvés au large de l'archipel espagnol a augmenté de moitié (Blanchard, Clochard et Rodier, 2008) ${ }^{30}$.

28 Cissé fait référence à l'histoire du repas. Cf. ci-dessus.

29 Comme il a été évoqué plus haut, les pêcheurs supportent généralement beaucoup mieux la traversée que ceux n'ayant aucune expérience de la mer.

30 Pour des données plus précises sur les morts aux frontières, cf. Clochard (2009). 


\section{L'ÉPREUVE DU REFOULEMENT}

Qu'ils soient partis par surprise ou que leur départ ait fait l'objet d'une concertation, les intéressés joignent généralement leur famille lorsqu'ils atteignent les îles Canaries. Lorsque leur embarcation est interceptée au large des côtes marocaines ou mauritaniennes, ils sont transférés dans des camps marocains ou mauritaniens avant d'être renvoyés au Sénégal par voie terrestre. Certains informent alors leurs proches par téléphone. Parmi ceux refoulés par charter des îles Canaries, certains avertissent leur famille une fois arrivés à l'aéroport de Dakar ou de Saint-Louis ${ }^{31}$. Mais partis sans avertir quiconque, nombreux sont ceux qui retournent chez eux par surprise. Il faut dire que ces voyages qui n'ont pas abouti suscitent des tensions au sein des rapports intra-familiaux mais aussi inter-familiaux à l'échelle du quartier. Des mères ont financé des voyages en utilisant des tontines, parfois à l'insu des autres participantes ${ }^{32}$, comptant sur le fait que leur fils rembourse la somme due une fois arrivé en Espagne. La faillite ainsi engendrée de ces circuits d'épargne féminins - et accentuée par les accusations d'abus de confiance retentit sur les rapports entre les mères et leur fils. Des refoulés s'établissent à l'écart de la maison familiale afin d'atténuer la pression des voisines qui exigent auprès de la mère un remboursement imminent.

\section{Solidarités collectives mises à mal : la confiance en question}

Aziz, mécanicien, est âgé d'une trentaine d'années. Lorsque je le rencontre, il est assis sur une natte, quelques boulons rouillés à ses côtés, sur la route de Yarakh. Avec lui, quelques camarades qui, eux aussi, ont effectué la traversée vers les îles Canaries. Marié et père de deux enfants de huit et deux ans, Aziz a vendu ses deux scooters et sa mère a emprunté la tontine de ses amies pour compléter le prix de la traversée. Il a tenté à deux reprises le voyage, mais les deux fois, a été refoulé. Cela fait plus d'un an maintenant qu'Aziz ne vit plus chez lui, mais avec des amis dans des petites guérites situées non loin de la plage. De temps à autre, sa femme et ses enfants viennent lui rendre visite, à moins qu'il ne se rende la nuit, en « cachette » dans la maison familiale. Mais, depuis son refoulement, sa mère préfère qu'il se tienne éloigné et ne se montre pas trop dans le quartier, pour apaiser les conflits avec les membres de la tontine.

Dans l'impossibilité de rembourser les dettes qu'ils ont pu contracter en leur nom propre, d'autres refoulés sont dénoncés à la police par leur "créancier ». En de telles circonstances, des compromis sont généralement trouvés à partir de l'établissement, sous contrôle judiciaire, d'un échéancier de remboursement. D'autres, qui pour financer leur propre voyage, ont endossé le rôle de rabatteur, sont dénoncés par des connaissances du quartier. Ces délations révèlent les limites de la solidarité collective. Désigner des coupables constitue, dans une double dynamique, un moyen de se préserver d'éventuelles mises en cause. Les refoulés mettent d'ailleurs en avant un ensemble de « pratiques de

31 Après les violentes manifestations ayant éclaté à Dakar suite aux premiers refoulements des îles Canaries, les charters en provenance des îles Canaries atterrissent à Saint-Louis, pour éviter des regroupements spontanés de refoulés qui se dispersent alors plus rapidement pour rentrer chez eux.

32 Parfois toutefois, il s'agissait de projets concertés au sein du groupe. 
justification » (Dubet, 1994 : 105) de leur tentative d'émigration. La rhétorique mobilisée emprunte à un double registre, marqué par un déni de responsabilité personnelle d'une part, et d'accusations portées à l'encontre de tiers d'autre part. Familles, rabatteurs, camarades leur ayant caché les conditions de la traversée, mais aussi Européens accusés de s'accaparer les ressources halieutiques du Sénégal ou encore de mener des politiques d'immigration instrumentalistes, sont autant d'acteurs qui, tenus pour responsables de leur émigration, les dédouanent, par effet domino, de leur acte. La construction des discours de victimisation, qui s'accompagne d'une logique de dédouanement, réfère ainsi à différents niveaux de réalité sociale allant de l'ordre micro-social à l'ordre politique.

Il n'en demeure pas moins que le retour à la maison familiale est à l'heure de règlements de compte. Certaines mères ou épouses, tenues à l'écart du projet du départ, se montrent fâchées à l'encontre de leur mari ou de leurs fils, même si elles sont généralement soulagées de le voir revenir en vie. Des conflits entre belles-familles éclatent, et parfois se soldent par des divorces. Là encore, c'est la rupture de confiance qui est mise en cause, même s'il serait réducteur d'attribuer aux seuls refoulements et départs non concertés, l'existence de tensions conjugales. Mais, si celles-ci préexistaient, elles n'en sont que plus exacerbées. C'est le cas de Modou dont la femme a demandé le divorce. Celle-ci n'était pas au courant du départ de son époux, contrairement à son beau-père et à sa belle-sœur. Des tensions traversent les familles lorsqu'un membre a financé la traversée à l'insu des autres proches, et que l'intéressé décède en mer ou demeure porté disparu. Mais ces distensions n'ont pas seulement une portée locale. Les refoulements s'accompagnent d'une exacerbation des procès à l'encontre des immigrés vivant en Europe et accusés de comportements individualistes. Nombre de refoulés leur reprochent de se complaire dans une consommation ostentatoire sans chercher à les soutenir. Ils remettent en cause la redéfinition des hiérarchies familiales qui tend à les minorer au profit de ces absents qui, pourtant, sont présents dans tous les esprits et érigés en modèles de réussite. Des non-dits deviennent explicites. En ayant misé sur quelqu'un d'autre, les familles qui ont participé au financement des traversées font savoir aux « mauvais » immigrés qu'ils ne remplissent pas leurs obligations. Il en est ainsi de la sœur de Modou. De plus, elle ne cache pas que son frère cadet, vivant en Italie depuis plusieurs années, est en froid avec elle depuis qu'il a appris qu'elle avait aidé Modou à partir en pirogues :

" J'ai essayé de lui expliquer, mais il ne m'écoute pas. Je lui ai dit que je n'avais pas 4 millions pour qu'il (Modou) parte en avion. Et lui, il n'a rien fait pour l'aider.

Il a répondu qu'il aide mon père, il veut l'aider, mais il n'a pas les moyens. Mais depuis il est fâché ».

En d'autres circonstances, toutefois, les membres de la famille immigrée ont pu être sollicités pour financer les traversées. Si ces sollicitations peuvent se faire à l'insu des proches au Sénégal, elles se font parfois à l'insu des émigrés eux-mêmes, ces derniers pensant que l'argent demandé est destiné à des frais d'urgence au Sénégal. Les refoulements s'accompagnent ainsi d'une mise à l'épreuve du lien social à travers un ébranlement des liens de solidarité et de confiance. 


\section{Héros, perdants, coupables ou victimes ? La position ambiguë des refoulés}

Il est courant que les refoulés soient, pour le sens commun, renvoyés à l'image d'individus marginalisés, disqualifiés dans l'espace social et familial. Certes, les refoulements des îles Canaries peuvent contribuer à accentuer une position de marginalisation sociale ; en d'autres circonstances, toutefois, ils s'accompagnent d'une certaine valorisation sociale, même si celle-ci demeure éphémère. Parce qu'elle resitue la dimension temporelle de l'action, la notion de trajectoire (Strauss, 1992a) permet de rendre compte de ces différentes périodisations (ou phasing) constitutives de l'expérience du refoulement ainsi que des rapports sociaux dans lesquels celle-ci se meut. L'exemple de Cissé est à cet égard éloquent. Lorsqu'il est refoulé au Sénégal, ce dernier attend la nuit pour regagner le domicile familial et ainsi, se soustraire au regard des voisins. Entre-temps, avec les quarante euros distribués par les autorités espagnoles ${ }^{33}$, il se rend au marché effectuer quelques achats pour les enfants de la maison, bref, autant de tentatives pour « sauver la face » (Goffman, 2005). Or, contre toute attente, il jouit, les premiers jours, d'une considération nouvelle au sein de sa famille :

"Mais le rapatriement, ça nous a aidés aussi. Car eux, ils ont commencé à connaître que nous, on veut quelque chose. Parce que moi, je pourrais me lever un jour et prendre le métier de menuisier, et je vais travailler. Mais pourquoi je ne le fais pas? Parce que... je suis dans un certain niveau, et je ne veux pas me réveiller pour aller apprendre un métier, c'est trop tard. J'ai fait des études. Mais, les gens

n'arrivent pas à comprendre. [...] Eux, ils ne croient pas aux études. Ils croient seulement à l'Europe. Quand j'étais dans les îles, ils me téléphonent chaque jour. Et comment ils parlent avec moi... ils me parlaient jamais comme ça avant! Ils sont en train de me considérer... Moi je me suis dit, c'est grave... l'homme n'est pas bon... je ne les reconnaissais plus. [...] Au moment que je reviens, ils me regardent encore bien. Ils pensent que tu as de l'argent, car tu as fait l'Europe un mois, et ils pensent que tu as fait quelque chose là-bas! Ils pensent que les Espagnols nous ont donné 400 euros! Au début, le retour ça a changé ça... pendant une ou deux semaines... Mais après ça recommence. Au début, tu es revenu et tu as un peu changé... Parce que... quand nous étions là-bas, dans le camp, on ne fait rien, on mange seulement et on se couche... donc tu as commencé à grossir... et il y a un complexe... Eux, ils doutent même, se demandent si tu n'as pas ramené un peu d'argent. Mais à la fin, ils comprennent que c'est le même Cissé et ils recommencent à parler sur toi... ».

Bien que Cissé ait été refoulé, sa tentative de rejoindre l'Europe marque un processus de « réajustement et d'alignement des cadres » (Snow, 2001) entre ses ambitions et celles de sa famille. Alors que ses proches n'accordent aucune valeur à la poursuite de ses études supérieures, perçues comme un investissement à perte ${ }^{34}$, en revanche, les perspectives de promotion sociale offertes par l'Europe font figure de représentations sociales

33 Lors des premiers refoulements, les autorités espagnoles remettent aux refoulés une enveloppe avec un peu d'argent en leur recommandant, d'après les témoignages recueillis, de ne pas la montrer aux autorités sénégalaises à leur arrivée à l'aéroport.

34 Antoine (2007) souligne à cet égard le sous-emploi fréquent des diplômés par rapport à leur niveau de qualification. De plus, comme nous l'avons précédemment souligné, ces derniers ne sont pas pour autant assurés d'échapper au chômage. 
partagées. Le regard valorisant que la famille de Cissé porte sur lui est aussi alimenté par sa méconnaissance des conditions de détention aux îles Canaries. Ce regard connaît toutefois une durée limitée, subordonnée au processus « d'alignement des cadres ». Lorsque, le temps passant, s'opèrent un effritement puis une " rupture de cadres », ses proches remettant en cause ses espoirs placés en lui, la marginalisation sociale que Cissé vivait dans sa famille reprend le dessus. À nouveau, il cherche à se dérober aux regards intransigeants qui l'accusent de " chômer ». Il retrouve ses amis dans sa chambre, cherchant à se faire oublier et à prendre de la distance vis-à-vis des commérages quotidiens portés à l'encontre de ceux qui ne soutiennent pas financièrement le foyer.

Si les regards portés sur les refoulés peuvent donc évoluer au cours du temps, une double étiquette sociale, celle de héros et de perdant, leur est également fréquemment accolée. Ce double étiquetage peut se conjuguer de manière simultanée et, dès lors, il correspond moins (comme précédemment) à un découpage en termes de "phasing » ou de paliers dans le temps, qu'il n'exprime l'ubiquité des identités sociales. À cet égard, la référence aux théories de l'étiquetage, que ce soit dans la tradition de l'École de Chicago (Becker, 1985) ou dans la lignée des théories modifiées (Lacaze, 2008) 35 $^{35}$, permet de mettre l'accent, dans une perspective constructiviste, sur la dynamique relationnelle de la considération et de la stigmatisation.

Les refoulés peuvent, dans un cercle de relations sociales données, faire l'objet d'une héroïsation : la sœur de Modou, par exemple, qualifie à plusieurs reprises son frère de " guerrier ». Initié aux pratiques maraboutiques, ce dernier a conseillé ses amis de Yarakh sur l'attitude à adopter avant la traversée (respect des prières, absence de relations sexuelles avec les filles de Saint-Louis qui venaient leur rendre visite dans la maison où ils attendaient l'annonce du départ, etc.). Aujourd'hui, Modou est très respecté à Yarakh, ses camarades gardant en mémoire sa position de « guide spirituel » durant le voyage. Lorsqu'il se rend dans le quartier, nombreux sont les jeunes qui le saluent avec considération. Sa femme, en revanche, a demandé le divorce tandis que le propriétaire de la chambre qu'il loue à Pikine, ne cache pas son mépris : Modou n'est pour lui qu'un refoulé de plus, qui a démissionné de son emploi pour se laisser berner par le mythe de l'Europe ${ }^{36}$. Ces positions hybrides, de surcroît, peuvent s'accompagner de situations plus ou moins difficiles à gérer.

De nombreux refoulés, par exemple, adoptent des stratégies d'évitement à l'égard des familles de camarades ayant péri en mer. À la douleur et au traumatisme d'avoir perdu un ami plus ou moins proche, peut s'ajouter la crainte d'être tenu responsable de ces décès, voire qu'une plainte ne soit portée à leur encontre. Le plus souvent, les intéressés expli-

35 La théorie de l'étiquetage modifiée, qui émerge dans les années 1980 dans le champ de l'analyse des troubles mentaux, met l'accent sur les processus d'auto-étiquetage. Elle insiste sur le fait qu'avant même d'être stigmatisé par d'autres, un individu peut avoir intériorisé une image négative de ce que le potentiel stigmate représente.

36 Au-delà des positions personnelles ainsi exprimées, les propos accusateurs tenus par des personnes tiers à l'égard des refoulés peuvent être influencés par la situation d'enquête, ce qui invite à rappeler l'importance complémentaire des observations et des récits croisés pour déjouer ces pièges méthodologiques. Comme le souligne Schwartz (1993), des enquêtés peuvent ajuster leurs discours en vue de faire « alliance » avec l'enquêteur et, ainsi, chercher à valoriser leur propre position. 
quent avoir perdu la trace de leur ami une fois arrivé dans l'archipel espagnol, à moins qu'ils ne prétendent avoir emprunté des embarcations différentes. De nombreuses familles sont ainsi laissées dans l'incertitude et se raccrochent à l'espoir, qui s'effile au cours du temps, de recevoir un improbable signe de vie de leur fils, de leur frère ou de leur mari. Mais les stratégies d'évitement ne sont pas toujours tenables sur la durée et la confrontation avec les proches des défunts est souvent inévitable. Mustapha a effectué la traversée avec son meilleur ami, décédé au cours du voyage. À son retour, il cherche à éviter son épouse, mais aussi les regards pesants du voisinage.

"Je sortais seulement le soir, pour qu'on ne me voit pas. Car je ne veux pas voir sa femme, qui pleure beaucoup. Mais avant, je suis resté deux mois sans sortir, découragé. Je ne pouvais pas sortir, je rêvais d'être en Europe, et je ne voulais pas croiser la femme de mon ami, c'est trop dur pour moi. Et il y avait encore d'autres problèmes à cause de la tontine de ma mère... La femme de mon ami vient me voir, mais mère lui dit à chaque fois que je dors. Un jour, la femme est entrée de force. Ma mère l'a laissée. On a discuté petit à petit... J'ai essayé de la réconforter... Grâce à Dieu. Après les deux mois, quand la femme est calmée, je suis parti chercher du travail. Car je suis parti avec un ami, il est mort, moi je ne pouvais pas continuer à vivre et à marcher dans le quartier... Pour moi, c'était bien de partir à Saly».

En dépit de ces situations inconfortables, voire douloureuses, nombre de refoulés rencontrés font état, en se remémorant leur expérience, d'une certaine fierté à avoir emprunté les «cayucos ». Leur expérience, mise en récit devant le chercheur, fédère même de manière tangible - un sentiment d'appartenance à un groupe, fondé sur une expression de la virilité, des ambitions partagées et la confrontation à des épreuves communes ${ }^{37}$. En ce sens, elle instaure une frontière entre des «Eux » (ceux qui n'ont pas tenté l'expérience) et des « Nous ». L'expérience de la migration, même avortée, est constitutive d'une forme de subjectivation (Dubet, 1994) qui s'accompagne de processus d'identification autour d'enjeux et de défis communs.

Bref, la manière dont les refoulés se considèrent et sont considérés par leur famille et le voisinage ne peut être figée dans une catégorisation rigide, mais nécessite d'être appréhendée dans une perspective attentive aux logiques « d'action située » (De Fornel et Quéré, 1999) et de " dynamiques de situation » (Bessin et Roulleau-Berger, 2002) où se conjuguent divers registres de rapports sociaux, se déclinant sur différentes échelles de temporalités et spatialités. Un refoulé peut être valorisé par les uns et dévalorisé par les autres, la construction de ces images positives et négatives variant dans le temps et dans l'espace.

\section{Les refoulements : entre rupture biographique et étape de parcours}

La littérature sociologique se rapportant à l'étude des parcours de vie se situe généralement dans une double perspective diachronique et chronologique (Bertaux,

37 Les discours recueillis comportent nécessairement, comme tout récit de trajectoire, une part de reconstruction pour donner du sens à une expérience et, qui plus est, à une expérience ayant tourné court (Bourdieu, 1986 ; Strauss, 1992a). Cette mise en cohérence biographique, qui fait partie intégrante de l'analyse sociologique, peut être considérée comme étant constitutive de l'expérience du refoulement. 
2003 ; Demazière et Dubar, 2004). Analysant les processus de continuité et de rupture biographiques (Grossetti, 2006), les travaux réalisés sont attentifs à la manière dont des évènements marquants ou turning-point (Heraldot, 2006) réorientent de manière plus ou moins imprévisible (Grossetti, 2004) les trajectoires de vie dans leur acceptation individuelle et collective. Dans le prolongement de ces perspectives théoriques, il est intéressant d'examiner la manière dont les refoulements des îles Canaries vers le Sénégal marquent, au-delà des simples parcours migratoires, les trajectoires biographiques des refoulés. De manière typologique, l'expérience du refoulement entrouvre sur deux grandes catégories de situation : le refoulement comme rupture et le refoulement comme revers de parcours ${ }^{38}$.

Dans un premier cas, en effet, le refoulement est vécu comme une rupture par rapport à la situation antérieure et par rapport au projet migratoire. Il en est ainsi pour des refoulés qui ont vendu leur matériel de travail pour financer la traversée ou dont les familles se sont lourdement endettées. C'est aussi le cas de refoulés qui, pour partir, ont démissionné de leur emploi sans pouvoir, par la suite, retrouver leur place professionnelle ${ }^{39}$. Pape qui, auparavant, réparait des téléphones portables d'occasion sur le marché Sandaga à Dakar, fait part de son désarroi :

"Pendant le rapatriement, là, je ne savais plus quoi penser. Quoi faire... J'étais là, comme le mur... Mais j'ai dit que j'allais laisser le Bon Dieu... En rentrant, [...] je peux dire que je n'ai pas dormi, je n'ai pas mangé... J'étais trop dégoûté, je ne voulais rien. Fumer seulement... J'enchainais les cigarettes... C'est depuis cette période-là même que je fume trop... Parfois même je ne mange pas... D'un côté, tu peux te réjouir, parce que tu pouvais rester en pleine mer, tu pouvais mourir... D'autre part, il y a une déception parce que tu voulais rentrer, travailler et aider ta famille et tu ne l'as pas eue. [...] Tu n'arrives pas à t'équilibrer dans ces deux pensées-là, c'est ça le problème. Et tu es revenu à la case départ. Je dirais que c'est même pire que de revenir à la case départ. [...] C'est une catastrophe car tu n'as plus rien! Ta famille n'a plus rien! [...] Bon le moral est cassé, mais tu sais, on croit en Dieu. [...] J'ai mis six mois avant de reprendre mon activité, avant je ne pouvais rien faire... Oui, six mois et deux semaines [soupirs]. Je ne pouvais parler à personne... [...] Après, je me suis dit qu'il fallait que je rattrape le temps perdu, que j'essaie de reprendre un travail ».

Le refoulement et l'échec du projet migratoire dépossèdent Pape de tout nouveau projet pendant un temps. Dans ce type de situation, les premiers jours, les premières semaines, voire les premiers mois suivant le refoulement, sont associés à la maladie, au stress (consommation excessive de cigarettes), à la dépression, voire à l'adoption de comportements déviants (consommation d'alcool, haschich). Ce n'est qu'au bout de plusieurs mois seulement, que les refoulés reprennent progressivement leurs activités quotidiennes, y compris parfois lorsqu'ils sont pères de famille.

Mamadou, quant à lui, a vingt-six ans lorsqu'il tente la traversée vers les îles Canaries. Il est alors marié, sans enfant. Après avoir obtenu un BTS gestion des entre-

38 Ces deux idéaux-types se retrouvent également dans l'expérience des Sénégalais rapatriés du Maroc vers le Sénégal au lendemain des évènements de Ceuta et Melilla en 2005 (Pian, 2010).

39 Ces situations de rupture se retrouvent également pour des refoulés sans emploi au moment du départ. 
prises et management ${ }^{40}$, il a travaillé deux ans en tant qu'assistant commercial, puis s'est retrouvé au chômage. Peu de temps après, son père décède. Lorsque Mamadou décide de prendre la mer, seul un frère aîné vivant à Paris est au courant de son intention. Son embarcation fait cependant naufrage au large des côtes mauritaniennes. Interné un mois durant dans un camp en Mauritanie, il est ensuite reconduit à la frontière du Sénégal puis transféré, inconscient, à l'hôpital de Dakar. Pour Mamadou, les traumatismes physiques et psychiques suscités par la traversée marquent tout autant la situation de rupture que les conséquences matérielles, financières et sociales du refoulement. À sa sortie de l'hôpital, il fait part de ses relations difficiles avec ses proches qui ne comprennent pas son geste :

"C'est comme si j'étais devenu un fardeau. [...] J'étais très malade et ma famille a dû décaisser beaucoup d'argent pour les frais médicaux. [...] Certains ont commencé à monter ma femme contre moi... Vraiment le retour c'était dur... J'avais perdu beaucoup de kilos, je ne ressemblais à plus rien du tout. Donc ce n'était plus moi. Une de mes tantes pleurait à chaque fois qu'elle me voyait. C'était comme si j'étais sur un lit de mort. [...] J'ai eu un sentiment de satisfaction un peu partout là où j'allais. Tu comprends? Tout le monde disait la même chose sur moi, que je suis poli, que j'aime les études, que tôt ou tard je vais réussir dans la vie... Et là, ce n'était plus l'image qu'ils avaient de moi. Il y en a même qui m'ont traité de fou... il y a beaucoup de choses qui peuvent te blesser ».

Le discours de Mamadou, qui porte un regard rétrospectif sur son parcours ${ }^{41}$, est structuré par un ensemble d'oppositions qui marquent la redéfinition de la considération portée sur lui avant et après sa tentative de rejoindre les îles Canaries. « L'avant »renvoyant à « l'agir intentionnel » de Schutz - est associé à l'espoir et à un statut valorisé ; « l'après », en revanche - renvoyant à l'action achevée -, est associé à la mort, «à rien », à la dégradation physique et à la disqualification sociale. Le modèle théorique de Dubar (1991) est alors éclairant. Les identités sociales, rappellent l'auteur, se construisent à la jonction d'une double dynamique où s'articule une « identité pour soi » (auto-revendiquée) et une « identité pour autrui » (attribuée de l'extérieur). La première, également appelée « transaction biographique » par Caradec (1998), renvoie à une " attitude réflexive qui vise à établir la continuité et la cohérence avec soi »; la seconde participe d'une « transaction relationnelle » où entre en jeu l'image que les « autruis », et notamment les proches, renvoient en miroir. Ces deux définitions, en constante interrelation, peuvent être plus ou moins conflictuelles, ou du moins en décalage. Elles peuvent être marquées par des continuités ou des discontinuités. Pour Mamadou, le refoulement marque une double rupture, touchant à la fois « l'identité pour soi » et « l'identité pour autrui », sa famille lui renvoyant, pour la première fois, une image négative de lui-même. L'expérience de la migration avortée, qui remet en cause l'équilibre des "identifications contingentes» (Dubar, 1991), représente ainsi un " incident critique » au sens où l'entend Strauss (1992b : 99), et qui constitue un « des moments décisifs dans le déroulement de la vie et de la carrière d'une personne [...] qui obligent à reconnaître que [l'on est] plus comme avant».

40 Lorsqu'il était étudiant, il dépose une demande de visa étudiant qui lui est refusé. Il en garde un profond sentiment d'injustice, d'autant plus que certains de ses amis ont eu en revanche une réponse positive.

41 L'on voit également ici comment son départ vers les îles Canaries s'effectue dans un moment où sa trajectoire biographique est marquée par certaines ruptures (décès du père, perte d'emploi). 
Dans un second cas, le refoulement n'est pas tant vécu comme une rupture, mais plutôt comme une étape de parcours. Ici, les refoulés reprennent au terme de quelques jours l'activité exercée avant le départ, à l'image de plusieurs pêcheurs et mareyeurs rencontrés à Mbour et rappelés par leurs responsabilités familiales. Pour autant la page de l'Europe n'est pas nécessairement tournée et, quelques semaines plus tard - entre dix jours et trois mois le plus souvent - certains tentent une nouvelle fois de rejoindre les îles Canaries. Leur détermination, malgré les risques dont ils sont désormais conscients, est entretenue par la conjugaison de plusieurs dimensions. Il y a, tout d'abord, les exemples - dont tout le monde parle dans le quartier - de connaissances transférées en Espagne à partir de l'archipel espagnol et qui, lors de la Tabaski ${ }^{42}$ notamment, commencent à envoyer des fonds à leur famille. Viennent ensuite les propos réconfortants que leur ont adressés des humanitaires espagnols lors de leur interception au large de l'archipel ou de leur séjour en centre de rétention. Comme le souligne Coureau (2007), l'action humanitaire est confrontée à une position ambiguë et, dans le contexte de contrôle des flux migratoires, cette ambiguïté n'échappe pas à la règle. Citant Brauman (2006), Coureau résume ainsi les dilemmes qui lui sont intrinsèques : "Qu'est-il mieux de faire : ne pas venir en aide et provoquer de terribles souffrances ou venir en aide et redonner de l'énergie aux combattants?». Cette citation prend également tout son sens dans le contexte des refoulements des îles Canaries vers le Sénégal.

À cela s'ajoute le besoin de main-d'œuvre dont l'économie espagnole - comme celle d'autres pays européens - est nécessiteuse, notamment parmi certains secteurs (agriculture, bâtiment, etc.) où les immigrés sans-papiers trouvent facilement à s'employer. Cette réalité économique, que reconnaît la représentation espagnole à Dakar, leur aurait été rappelée par des humanitaires espagnols ou des membres de la Garda civil leur ayant manifesté une forme de compassion. Si ces dimensions s'agrègent pour encourager les refoulés à tenter une seconde, voire une troisième fois, la traversée vers les îles Canaries, ces tentatives successives sont matériellement rendues possibles par la structuration des réseaux migratoires qui, comme nous l'avons mentionné, permet aux pêcheurs de se reconvertir en capitaine de pirogue, sans alors à avoir à payer la traversée. Les réseaux de connaissance permettent également aux habitants du littoral d'obtenir des tarifs avantageux, parfois divisés de plus de moitié ${ }^{43}$. Et, pour ceux qui ne peuvent prétendre à ces tarifs préférentiels, des solutions alternatives peuvent être envisagées. Comme cela a été le cas pour Cissé, les départs par "forcing » où les intéressés, renseignés sur le lieu et la date d'embarquement, attendent à l'affût pour se joindre au voyage, constituent des cas fréquents qui donnent lieu à des rixes parfois violentes ${ }^{44}$. Mais la crainte suscitée par l'intervention de la police contraint souvent les capitaines à accepter à bord quelques passagers supplémentaires.

42 Fête musulmane couramment appelée « fête du mouton ».

43 Des situations différentes seraient sans doute observées auprès des refoulés originaires de l'intérieur des terres, l'organisation même du voyage ne mobilisant pas nécessairement les mêmes contraintes ni ressources (possibilité de faire partie de l'équipage, voire d'être capitaine de pirogue, etc.).

44 Afin de déjouer les contrôles policiers, les départs s'effectuent en deux temps. Les passagers embarquent à bord d'une première pirogue de taille moyenne qui les achemine par groupes vers le « cayucos », arraisonné en haute mer, dans lequel s'effectuera la traversée vers les îles Canaries. 
Entre ces deux volets typologiques - le refoulement comme rupture et le refoulement comme étape de parcours - se donnent à lire des situations intermédiaires aux contours flexibles. Les situations de rupture, de surcroît, peuvent s'accompagner d'un engrenage qui encourage à tenter un second, voire un troisième voyage. C'est le cas de Mamadou qui, deux mois après être sorti de l'hôpital, prépare une nouvelle traversée :

"C'est ce goût d'inachevé qui m'a poussé à repartir. Après la guérison, je me disais voilà, il faut que je reparte. À cause de la famille, des regards... tu sais, au Sénégal, on dit que la parole c'est comme une balle. Quand ça sort c'est fini. Elle ne peut plus revenir en arrière. Il y a des regards qui m'ont blessé ».

Pour financer son second voyage, qui se soldera par un nouveau refoulement, Mamadou met à profit son expérience acquise en endossant le rôle de rabatteur.

Il est rare, cependant, que des refoulés tentent plus de trois fois de suite la traversée vers les îles Canaries. Le voyage à tout prix vers l'Europe a ses limites. Outre les contraintes financières et matérielles, les superstitions populaires invitent à ne pas « forcer le destin $»^{45}$. Pour autant, leur rêve de l'Europe n'est toujours pas dissipé. Recevant parfois des appels téléphoniques de leurs amis parvenus en Espagne, voire un peu d'argent de leur part, certains refoulés confient qu'ils préfèrent dépenser la somme reçue en "une soirée, en se comportant comme des toubab en boite de nuit » plutôt que de chercher à économiser sur un montant qui leur semble dérisoire au regard d'un avenir sans projection.

\section{CONCLUSION}

Après une forte médiatisation en 2006-2008, les traversées clandestines vers les îles Canaries depuis le littoral sénégalais se sont progressivement taries, tandis que l'externalisation de la lutte contre l'immigration clandestine, à travers des dispositifs de contrôles tels que Frontex (agence européenne pour la gestion de la coopération opérationnelle aux frontières extérieures) et les systèmes de surveillance SIVE (Système Intégré de Vigilance Extérieure), n'ont cessé de se renforcer entre les côtes canariennes et sénégalaises ${ }^{46}$. En dépit de son caractère, somme toute relativement éphémère, le " phénomène des pirogues » et les refoulements au Sénégal qui l'ont accompagné, ont joué comme des révélateurs de tensions familiales. Pour en saisir toute la portée, il semblait nécessaire d'appréhender ces rapports sociaux dans la déclinaison de leurs dimensions temporelles et spatiales, avant les départs et après les refoulements. Cette perspective invite à rappeler un principe méthodologique de base selon lequel les conditions du retour ne peuvent être analysées indépendamment des conditions du départ (Sayad, 1999), et pouvons-nous ajouter, des conditions du voyage et du passage des frontières. Enfin, au-delà de son impact sur les formes de

45 En outre, il convient de faire une distinction entre les projets de nouvelles tentatives de traversée vers les îles Canaries et leur mise en acte. Entre nos deux terrains menés à environ quatre mois d'intervalle, nous avons ainsi pu constater une évolution des projets des refoulés et certains d'entre eux qui, en février-mars, se disaient prêts à repartir à la moindre occasion, tenaient des propos beaucoup plus nuancés au mois de juillet. L'analyse longitudinale des trajectoires nécessite donc de prendre en compte deux niveaux de réalité sociale distincts.

46 Ces dispositifs de surveillance sont également à l'œuvre au large des côtes marocaines et mauritaniennes notamment. 
sociabilités locales, il importe de souligner que le « phénomène des pirogues » a accru la perte de confiance d'une partie de la population sénégalaise à l'égard du gouvernement sénégalais, doublement mis en cause dans sa gestion de la question migratoire. D'une part, l'envoi de la DIC sénégalaise (Direction d'Investigation Criminelle) aux îles Canaries afin d'organiser les refoulements est perçu comme une forme de trahison; d'autre part, l'inertie de l'État dans la mise en place de programmes de réinsertion au profit des refoulés a décrédibilisé un peu plus l'action des autorités ${ }^{47}$. À cela s'ajoutent les procès très controversés pour " émigration illégale $»^{48}$ qui ont eu lieu au Sénégal, et qui révèlent d'autant l'incohérence de la politique menée par le gouvernement ou, plutôt, sa récupération de la question migratoire à des fins de politique interne. Car d'un côté, certains refoulés sont déférés devant la justice ; de l'autre, le président sénégalais, alors en pleine campagne électorale, annonce qu'ils seront les bénéficiaires privilégiés des contrats de travail saisonniers espagnols négociés avec l'Espagne ${ }^{49}$. Mais, très vite, le gouvernement revient sur ces déclarations très médiatiques. En effet, dès lors que les refoulés ont été fichés au niveau du fichier Schengen, ils ne peuvent plus se rendre légalement en Europe durant un certain temps. Pour tenter de sauver la mise, les autorités sénégalaises leur proposent alors, en cas de sélection, de choisir un membre de leur famille pour partir à leur place... Il ne va pas sans dire que cela attise à nouveau des tensions familiales...

\section{Références bibliographiques}

ANTOINE Philippe (Éd.) (2007) Les relations intergénérationnelles en Afrique : approche plurielle, Paris, Ceped, 255 p.

ANTOINE Philippe (2007) La place et l'activité des personnes âgées dans sept capitales ouestafricaines, in Philippe Antoine Éd., Les relations intergénérationnelles en Afrique : approche plurielle, Paris, Ceped, pp. 31-62.

ANTOINE Philippe, BOCQUIER Philippe, FALL Abdou Salam, GUISSE Youssouf M. et NANITELAMIO Jeanne (1995) Les familles dakaroises face à la crise, Paris, Orstom/Ceped/ Ifan, $209 \mathrm{p}$.

ANTOINE Philippe et NANITELAMIO Jeanne (1995) Peut-on échapper à la polygamie à Dakar?, Paris, Ceped, $31 \mathrm{p}$.

BEAUD Stéphane et WEBER Florence (2003) Guide de l'enquête de terrain, Paris, La Découverte, $356 \mathrm{p}$.

47 Cf. notamment les polémiques à propos du plan Reva (Pian, 2009b).

48 Comme le souligne Morice (2009), la notion « d'émigration illégale » apparaît en porte-à-faux avec la Déclaration universelle des droits de l'Homme (1948) qui, dans son article 13, conçoit « le droit de quitter son pays » (et d'y revenir) comme un droit minimal. Les Sénégalais interceptés au large des côtes sénégalaises ou refoulés des îles Canaries, et déférés devant la justice au Sénégal, sont généralement relaxés après de sévères mises en garde, s'ils n'ont pas participé à l'organisation des traversées. Dans le cas contraire, ils risquent plusieurs mois de prison et une amende. Pour donner quelques chiffres, du 6 septembre 2006 au 19 juin 2007, 2056 personnes ont été interpellées au Sénégal, dont 1702 arrêtées en mer, 756 à terre et 48 « convoyeurs » (Source : OIM, Bulletin d'information, 3, juin 2007).

49 À Mbour, un pêcheur ayant purgé six mois de prison pour avoir participé à l'organisation d'un voyage clandestin vers les îles Canaries, a ainsi obtenu un contrat de travail saisonnier pour l'Espagne alors même qu'il était encore en détention à la prison de Thiès ! 
BECKER S. Howard (1985) Outsiders. Études de sociologie de la déviance, Métailié, Paris, 247 p. BERTAUX Daniel (2003) Les récits de vie. Perspective ethnosociologique, Paris, Nathan, 128 p.

BESSIN Marc et ROULLEAU-BERGER Laurence (2002) Les armes du faible sont-elles de faibles armes ?, L'Homme et la société, 143-144, pp. 3-11.

BLANCHARD Emmanuel, CLOCHARD Olivier et RODIER Claire (2008) Compter les morts, Plein droit, 77, pp. 30-33.

BOUILLY Emmanuelle (2008) Les enjeux féminins de la migration masculine : le Collectif des femmes pour la lutte contre l'immigration clandestine de Thiaroye-Sur-Mer, Politique Africaine, 109, pp. 16-31.

BRAUMAN Rony (2006) Préface, in Karl Blanchet et Boris Martin Éds., Critique de la raison humanitaire, Le cavalier bleu, Paris, pp. 5-11.

CARADEC Vincent (1998) Les transitions biographiques, étapes du vieillissement, Prévenir, 35, 2e sem., pp. 131-137.

CEFAÏ Daniel (1994) Type, typicalité, typification, in Bernard Fradin, Louis Quéré et Jean Widmer Éds., L'enquête sur les catégories. De Durkheim à Sacks, Paris, EHESS, pp. 105-128.

CHARBIT Yves et KEBE Mamabou (2007) Genre et vulnérabilité au Sénégal : les femmes chefs de ménage, Revue Européenne des Migrations Internationales, 23 (3), pp. 51-65.

CLOCHARD Olivier (2009) Morts aux frontières : les routes changent, mais les drames subsistent, in Olivier Clochard Éd., Atlas des migrants en Europe. Géographie critique des politiques migratoires, Paris, Armand Colin, pp. 116-118.

COUREAU Henri (2007) Ethnologie de la forme-camps de Sangatte. De la régulation à l'exception, Paris, Éditions des Archives contemporaines, 274 p.

DAFFE Gaye et DANSOKHO Mamadou (2003) Les nouvelles technologies de l'information et de la communication : défis et opportunités pour l'économie sénégalaise, in Momar-Cumba Diop Éd., Le Sénégal à l'heure de l'information, Paris, Karthala, pp. 45-96.

DAHOU Tarik et FOUCHER Vincent (2005) Le Sénégal, entre changement politique et révolution passive. « Sopi » or not « sopi »?, Politique africaine, 96, pp. 15-21.

DE FORNEL Michel et QUÉRÉ Louis (1999) La logique des situations. Nouveaux regards sur l'écologie des activités sociales, EHESS, Paris, 358 p.

DEMAZIĖRE Didier et DUBAR Claude (2004) Analyser les entretiens biographiques. L'exemple des récits d'insertion, Saint-Nicolas, Presses de l'Université Laval, 350 p.

DIAGNE Alioune et LESSAULT David (2007) Émancipation résidentielle et rapports intergénérationnels à Dakar, Paris, Ceped, 45 p.

DIAL Fatou Binetou (2007) Mariage et divorce à Dakar. Itinéraires féminins, Paris, Kartala, 182 p.

DIOP Momar-Coumba (2009) Mobilités, État et société, in Momar-Coumba Diop Éd., Le Sénégal des migrations, Paris, Karthala, pp. 13-35.

DUBAR Claude (1991) La socialisation, Paris, Armand Colin, 255 p.

DUBET François (1994) Sociologie de l'expérience, Paris, Seuil, 272 p.

FOUQUET Thomas (2009) Migrations et « glocalisation » dakaroises, in Momar-Cumba Diop Éd., Le Sénégal des migrations, Paris, Karthala, pp. 241-276.

GODENAU Dirk et HERNANDEZ Zapata V.M. (Eds.) (2007) La immigracion irregular en Tenerife, [en ligne] consulté le 6 février 2011. URL : http://www.obiten.net

GOFFMAN Erving (2005) Les rites d'interaction, Paris, Les Éditions de Minuit (1 1 ère traduction en français, 1974), $236 \mathrm{p}$.

GOFFMAN Erving (1991) Les cadres de l'expérience, Paris, Les Éditions de Minuit, 573 p.

GONIN Patrick et ROBIN Nelly (2009) Les routes migratoires par le Sénégal, in Ali Bensaad Éd., Le Maghreb à l'épreuve des migrations transsahariennes, Paris, Karthala, pp. 137-167.

GROSSETTI Michel (2006) L'imprévisible dans les parcours sociaux, Cahiers internationaux de sociologie, CXX, pp. 5-28.

GROSSETTI Michel (2004) Sociologie de l'imprévisible : dynamique de l'activité et des formes sociales, Paris, Puf, 255 p. 
GUÉRIN Isabelle (2008) L'argent des femmes pauvres : entre survie quotidienne, obligations familiales et normes sociales, Revue française de socio-économie, 2 (2), pp. 59-78.

HERLARDOT Valentine (2006) Parcours professionnels et histoire de santé : une analyse sous l'angle des bifurcations, Cahiers internationaux de sociologie, CXX, pp. 59-84.

JOUNIN Nicolas (2008) Chantier interdit au public : enquête parmi les travailleurs de bâtiment, Paris, La Découverte, 275 p.

LACAZE Lionel (2008) La théorie de l'étiquetage modifiée, ou « l'analyse stigmatique » revisitée, Nouvelle revue de psychologie, 1 (5), pp. 183-199.

MARIE Alain (Éd.) (1997) L'Afrique des individus, Paris, Karthala, 438 p.

MARRERO Alexis Mesa (Ed.) (2008) Guia pratica para la investigacion de la immigracion en la escala local. Observatorio de la immigracion en Tenerife, [en ligne] consulté le 6 février 2011. URL : http://www.obiten.net

MERTON Robert King (1953) Éléments de méthode sociologique (traduit De l'américain par Henri Mendras), Paris, Plon, 248 p.

MORICE Alain (2009) Conceptualisation des migrations et marchandages internationaux, in Ali Bensaad (Éd.), Le Maghreb à l'épreuve des migrations transsahariennes, Karthala, Paris, pp. 193-212.

PIAN Anaik (2011) The "discursive framework" of development and the repertoire of actions of senegalese deportee associations, in Jocelyne Streiff-Fenart and Aurélia Wa Kabwe Sagatti Eds., The challenge of the threshold. Border closures and migration movements in Africa, Maryland, Lexingtonbooks (sous presse).

PIAN Anaik (2010) Trajectoires de rapatriés. Éléments pour une réflexion en termes d'expérience, Hommes et migrations, 1286-1287, pp. 74-97.

PIAN Anaik (2009a) Aux nouvelles frontières de l'Europe. L'aventure incertaine des Sénégalais au Maroc, Paris, La Dispute, 237 p.

PIAN Anaik (2009 b) Face aux enjeux politiques locaux, aide au développement en échange de retours forcés : le Plan Reva au Sénégal, in Olivier Clochard Éd., Atlas des migrants en Europe. Géographie critique des politiques migratoires, Paris, Armand Colin, pp. 88-90.

SALL Aliou et MORAND Pierre (2008) Pêche artisanale et émigration des jeunes africains par voie piroguière, Politique Africaine, 109, pp. 32-41.

SAYAD Abdelmalek (1999) La double absence. Des illusions de l'émigré aux souffrances de l'immigré, Paris, Seuil, 437 p.

SCHUTZ Alfred (1994) Le chercheur et le quotidien, Paris, Meridien Klincksieck, 286 p.

SCHUTZ Alfred (1932) Der sinnhafte Aufbau der sozialen Welt. Eine Einleitung in die verstehende Soziologie, Vienne, Springer, 352 p.

SCHWARTZ Olivier (1993) L'empirisme irréductible, in Nels Anderson, Le Hobo, Paris, Nathan, pp. 265-308.

SNOW David (2001) Analyse de cadres et mouvements sociaux, in Daniel Cefaï et Danny Trom, Les formes de l'action collective. Mobilisations dans des arènes publiques, EHESS, Paris, pp. $27-50$.

STRAUSS Anselm (1992a) La trame de la négociation. Sociologie qualitative et interactionniste (textes réunis et présentés par Isabelle Baszanger), Paris, L'Harmattan, 311 p.

STRAUSS Anselm (1992 b) Miroirs et masques. Une introduction à l'interactionnisme, Paris, Métailié, 192 p. 


\title{
Migrations internationales au prisme des rapports familiaux. Les familles sénégalaises à l'épreuve des refoulements des îles Canaries
}

\author{
Anaik Pian
}

En 2006, tandis que les côtes sénégalaises deviennent un point de départ privilégié vers les îles Canaries, les Sénégalais qui tentent de rejoindre l'archipel espagnol en « cayucos » s'emparent du slogan suivant : « Barcelone ou la mort » (Barça ou Barzakh). Emblème de leur détermination à rejoindre l'Europe, cette expression qui s'est popularisée ne prend pourtant pas en compte une éventualité bien présente : celle de l'arrivée aux îles Canaries puis du refoulement par charter au Sénégal qui, pour l'année 2006, a concerné plus de 6000 Sénégalais. Quels sont les impacts de ces refoulements sur les rapports familiaux et, plus largement, sur la vie des quartiers très touchés par les départs en pirogues vers les îles Canaries ? Cet article s'intéresse ainsi à la dynamique des rapports familiaux qui ont entouré, en amont comme en aval, ce qui a été communément appelé le " phénomène des pirogues ». In fine, il propose une typologie de l'expérience du retour forcé pour les refoulés.

\section{International Migrations through the Prism of Family Relationships. Senegalese Families Difficult Experience of Migrants being Expelled from Canary Islands}

\begin{abstract}
Anaik Pian
In 2006, while senegalese shores are becoming a preferential starting-point towards Canary Islands, Senegalese migrants trying to reach the spanich archipelago in "cayucos", take hold of the following slogan: "Barcelona or death" (Barça or Barkazh). These words have become popular among migrants and symbolize their determination to reach Europe. However, they do not take into consideration this quite likely eventuality, which concerned more than 6,000 Senegalese migrants in 2006: being expelled back to Senegal by charter, after having reached Canariy Islands shores. What impacts do these driving back have on family relationships and more widely, on neighbourhood life in these districts much concerned by numerous departures in "cayucos" towards Canary Islands? This article takes an interest in the dynamics of family relationships wich have accompanied up-stream as well as downstream, what has been commonly called "the cayucos phenomenon". Lastly, it proposes a typology of expelled migrants forced return's experience.
\end{abstract}

\section{Migraciones internacionales desde la perspectiva de las relaciones familiares. Las familias senegalesas ante las devoluciones en frontera de las Islas Canarias}

\section{Anaik Pian}

En 2006, mientras que las costas senegalesas se convierten en un punto de salida privilegiado hacia las Islas Canarias, los senegaleses que intentan alcanzar el archipiélago español en «cayucos» hacen suyo el siguiente lema: «Barcelona o muerte» (Barça o Barkazh). Sin embargo, esta expresión popularizada, emblema de su determinación por alcanzar Europa, no tiene en cuenta una eventualidad muy real: la llegada a las islas Canarias y la consiguiente devolución por chárter a Senegal, que en el año 2006 afectó a más de 6000 senegaleses. ¿Qué impacto tienen estas devoluciones en frontera en las relaciones familiares y, más globalmente, en la vida de los barrios más afectados por las salidas en piroga hacia las Islas Canarias? Este artículo aborda la dinámica de las relaciones familiares que rodearon, de principio a fin, lo que comúnmente se ha llamado el «fenómeno de las pirogas». Finalmente, propone una tipología de la experiencia del retorno forzado de las personas devueltas en frontera. 